\title{
Phase I dose escalation study of telatinib (BAY 57-9352) in patients with advanced solid tumours
}

\author{
D Strumberg*, , B Schultheis', IA Adamietz ${ }^{2}$, O Christensen ${ }^{3}$, M Buechert $^{4}$, J Kraetzschmar ${ }^{5}$, P Rajagopalan ${ }^{3}$, \\ M Ludwig ${ }^{5}$, A Frost ${ }^{6}$, S Steinbild ${ }^{6}$, ME Scheulen ${ }^{7}$ and K Mross ${ }^{6}$ \\ 'Department of Haematology and Medical Oncology, University of Bochum (Marien Hospital, Herne), Herne, Germany; ${ }^{2}$ Department of Radiotherapy, \\ University of Bochum (Marien Hospital, Herne), Herne, Germany; ${ }^{3}$ Bayer Pharmaceuticals Inc., Montville, NJ, USA; ${ }^{4}$ Magnetic Resonance Development \\ and Application Centre, University Hospital, Freiburg, Germany; ${ }^{5}$ Bayer Schering Pharma, Wuppertal, Germany; ${ }^{6}$ Tumour Biology Centre at the Albert- \\ Ludwigs-University, Freiburg, Germany; ${ }^{7}$ Department of Internal Medicine and Medical Oncology, West German Cancer Centre, University of Essen, \\ Germany
}

Telatinib (BAY 57-9352) is an orally available, small-molecule inhibitor of vascular endothelial growth factor receptors 2 and 3 (VEGFR-2/-3) and platelet-derived growth factor receptor $\beta$ tyrosine kinases. In this multicentre phase I dose escalation study, 7I patients with refractory solid tumours were enroled into 14 days on/7 days off (noncontinuous dosing) or continuous dosing groups to receive telatinib two times daily (BID). Hypertension (23\%) and diarrhoea (7\%) were the most frequent study drug-related adverse events of CTC grade 3. The maximum-tolerated dose was not reached up to a dose of I 500 mg BID continuous dosing. Telatinib was rapidly absorbed with median $t_{\max }$ of 3 hours or less. Geometric mean $C_{\max }$ and $A \cup C_{0-12}$ increased in a less than doseproportional manner and plateaued in the $900-1500 \mathrm{mg}$ BID dose range. Two renal cell carcinoma patients reached a partial response. Tumour blood flow measured by contrast-enhanced magnetic resonance imaging and sVEGFR-2 plasma levels decreased with increasing $\mathrm{AUC}_{0-12}$ of telatinib. Telatinib is safe and well tolerated up to a dose of $1500 \mathrm{mg}$ BID continuous dosing. Based on pharmacokinetic and pharmacodynamic criteria, $900 \mathrm{mg}$ telatinib BID continuously administered was selected as the recommended phase II dose.

British Journal of Cancer (2008) 99, I579- 1585. doi:I0.1038/sj.bjc.6604724 www.bjcancer.com (c) 2008 Cancer Research UK

Keywords: phase I; telatinib; vascular endothelial growth factor receptor; tyrosine kinase inhibitor; pharmacodynamics; pharmacokinetics

Telatinib (BAY 57-9352) is an orally available, potent, smallmolecule inhibitor of vascular endothelial growth factor (VEGF) receptors 2 and 3 (VEGFR-2/-3) and platelet-derived growth factor (PDGF) receptor $\beta$ (PDGFR- $\beta$ ) tyrosine kinases.

The growth of solid tumours is accompanied by angiogenesis (Algire et al, 1945) and the development of an effective vascular network is required for the tumours to grow beyond $1 \mu \mathrm{l}$ in volume (Folkman, 1990). Anti-angiogenesis is an interesting strategy for the treatment of cancer (Folkman, 1971). VEGF and its receptor, VEGFR-2, which is expressed on activated endothelial cells associated with growing solid tumours, are required for the angiogenic process (Folkman, 2002; Pisacane and Risio, 2005). Continued signal transduction through the VEGF/VEGFR-2 pathway is a primary stimulus for initiation and maintenance of tumour angiogenesis (Ellis, 2004). Blocking the interaction of VEGF with the VEGFR-2 receptor or inhibiting the tyrosine kinase activity of the VEGFR-2 receptor blocks both angiogenesis and

\footnotetext{
* Correspondence: Dr D Strumberg; Department of Haematology and Medical Oncology, University of Bochum (Marien Hospital, Herne), Herne, Hoelkeskampring 40, 44625 Germany;

E-mail: dirk.strumberg@marienhospital-herne.de

Received 15 September 2008; accepted 16 September 2008
}

tumour growth in in vivo models. Complete suppression of tumour growth has been demonstrated using dominant-negative VEGF receptors (Zhang et al, 1995; Millauer et al, 1996; Goldman et al, 1998) and blocking antibodies (Kim et al, 1993; Asano et al, 1995; Warren et al, 1995; Melnyk et al, 1996; Yuan et al, 1996; Borgstrom et al, 1998) as well as small-molecule inhibitors of VEGFR-2 kinase (Wood et al, 2000; Wedge et al, 2002) as single-agent therapies in model systems. Overexpression of VEGF is common in solid tumours and associated with poorer prognosis (Dosquet et al, 1997; Raben and Helfrich, 2004; Giatromanolaki et al, 2006).

Telatinib is a potent inhibitor of VEGFR-2 and PDGFR- $\beta$ tyrosine kinase activity measured in a biochemical assay. These two receptors play key roles in the angiogenic process involving the stimulation of endothelial cells and PDGFR-expressing pericytes. Telatinib inhibited VEGFR-2 autophosphorylation in a whole-cell assay of receptor autophosphorylation in vitro, VEGF-dependent proliferation of human umbilical vein endothelial cells in vitro, and PDGF-stimulated growth of human aortic smooth muscle cells. Telatinib demonstrated potent, dose-dependent reduction in tumour growth in vivo in a variety of models including MDA-MB231 breast carcinoma, Colo-205 colon carcinoma, DLD-1 colon carcinoma and H460 non-small cell lung carcinoma. Toxicological studies supported the start of a clinical study in cancer patients at a dose level of $10 \mathrm{mg}$ (once daily (OD)) of telatinib. 
The N-methyl group of telatinib was identified as the main target of metabolic degradation. The in vitro investigations using human microsomes, hepatocytes or single cytochrome P450 (CYP) isoforms revealed that there is no or only a very low risk of drug-drug interactions. Telatinib was metabolised by various CYP isoforms. There was no critical involvement of polymorphic CYP isoforms in the biotransformation. Telatinib exhibited neither an inhibitory nor an inductive potential on major human CYP isoforms at therapeutically relevant concentrations. Drug-drug interactions are also unlikely to occur due to displacement from plasma protein-binding sites or modulation of p-glycoprotein transporter activity based on the results of in vitro studies.

This phase I clinical study had the objective to determine the dose-limiting toxicities (DLTs), maximum-tolerated dose (MTD) and pharmacokinetics of oral telatinib. Preliminary antitumour activity, interaction with a variety of biomarkers including VEGFR-2 and dynamic contrast-enhanced magnetic resonance imaging (DCE-MRI) were evaluated.

\section{PATIENTS AND METHODS}

\section{Patient selection}

Eligible patients were $\geqslant 18$ years of age, with a life expectancy of at least 12 weeks, and a solid tumour that was refractory to standard treatment or without standard therapy options. Patients had to have Eastern Cooperative Oncology Group (ECOG) performance status of $0-1$. All patients had evaluable disease according to the Response Evaluation Criteria in Solid Tumours (RECIST) criteria. Patients might have had any number of prior systemic therapy, radiotherapy or surgery, but therapies had to be discontinued at least 4 weeks before study entry (6 weeks in case of mitomycin $\mathrm{C}$ and nitrosoureas).

Other eligibility criteria included the following: (1) adequate haematopoietic (absolute neutrophil count (ANC) $\geqslant 1.5 \times 10^{9}$ $1^{-1}$; platelet count $\geqslant 150 \times 10^{9} 1^{-1}$ and haemoglobin $\geqslant 9.0 \mathrm{~g} \mathrm{dl}^{-1}$ ), hepatic (total bilirubin $\leqslant 1.5$ times the upper limit of normal (ULN); aspartate aminotransferase and alanine aminotransferase $\leqslant 2.5$ times ULN; prothrombin time and international normalised ratio of partial thromboplastin time $<1.5$ times ULN unless on therapeutic anticoagulants), and renal (serum creatinine $\leqslant 1.5$ times ULN) functions; (2) no pregnancy and breast feeding; (3) no clinically relevant co-morbidity such as cardiovascular diseases and no clinically relevant co-medication; (4) no metastatic brain or meningeal tumours, unless the patient was $>6$ months from definitive therapy and had a negative imaging study within 4 weeks of study entry.

All patients provided written informed consent in accordance with federal and institutional guidelines before study treatment.

\section{Study design}

This was a multicentre, open-label, non-controlled, phase I dose escalation study to investigate the safety, pharmacokinetics and pharmacodynamics of oral telatinib (BAY 57-9352). Administration of telatinib was continued until an unacceptable toxicity, disease progression or death occurred or the consent was withdrawn. At start of the study, only a solution formulation was available. The formulation as tablet was introduced into the study after first pharmacokinetic results became available. Based on pharmacokinetic data, OD, two times daily (BID), and three times daily schedules were evaluated. For the sake of clarity, the data presented in this paper refer to the patients enroled into the BID 14 days on/7 days off (noncontinuous dosing) and continuous dosing groups only.
Three patients were initially enroled at each dose level. If no DLT had occurred at the end of the 3-week treatment cycle, three patients were enroled at the next dose level. If any patient experienced a DLT, three additional patients were enroled at that dose level. If at least two out of six patients experienced a DLT, dose escalation had to be stopped and that dose was to be declared the toxic dose. The next lower dose level was defined as the MTD. DLTs were defined as grade 3 or 4 non-haematological toxicities, febrile neutropenia (ANC $<0.5 \times 10^{9} 1^{-1}$ and fever $\geqslant 38.5^{\circ} \mathrm{C}$ ), grade 4 neutropenia lasting for at least 7 days, platelet count $<25 \times 10^{9} 1^{-1}$ or grade 3 or 4 thrombocytopaenic bleeding, which occurred during cycle 1 . In the course of the study the protocol was amended so that the onset of CTC grade 3 hypertension was only considered to be a DLT if the hypertension turned out to be refractory to standard antihypertensive treatment. The number of patients enroled per dose level was extended to six patients for the dose levels of $150 \mathrm{mg}$ BID or higher to get more reliable estimates for telatinib pharmacokinetic parameters.

Adverse events were assessed at the end of each cycle and graded according to the National Cancer Institute Common Toxicity Criteria (NCI CTC), v2.0 (Trotti et al, 2000).

\section{Patient evaluation}

History, physical examinations, haematological and biochemical laboratory evaluations were performed at screening, on days 1, 7 and 14 of cycle 1 and on day 1 of subsequent cycles. Baseline objective tumour measurements were performed within 4 weeks prior to study treatment. Lesions at all disease sites were categorised as either measurable or nonmeasurable. Indicator lesions were selected and monitored throughout the study by the same assessor and using the same technique. Tumour response was evaluated according to the RECIST (Therasse et al, 2000).

\section{Pharmacokinetics}

Patients with at least one valid pharmacokinetic profile were valid for the pharmacokinetic analysis. Plasma samples were collected at predose and $0.5,1,2,3,4,6,8$, and $12 \mathrm{~h}$ postdose on day 1 and day 14 of cycle 1 and were analysed for BAY 57-9352 and its demethylated metabolite M-2, BAY 60-8246, using a validated LC-MS-MS analytical method.

Plasma pharmacokinetic parameters, area under the curve from time $0-12 \mathrm{~h}$ after dosing $\left(\mathrm{AUC}_{0-12}\right)$, area under the curve from time 0 to last data point $\left(\mathrm{AUC}_{0-\mathrm{tn}}\right)$, maximum plasma concentration $\left(C_{\max }\right)$, and time to maximum plasma concentration $\left(t_{\max }\right)$ of telatinib and its metabolite (BAY $60-8246)$ as well as half-life of telatinib were calculated by non-compartmental methods using WinNonlin version 4.1.a (Pharsight Corporation). The linearlogarithmic trapezoidal rule was used for calculating AUC. Half-life was calculated by linear least squares regression after logarithmic transformation of the terminal concentrations. Pharmacokinetic parameters were analysed using descriptive statistics.

\section{Pharmacodynamics}

The effects of telatinib treatment on the plasma concentrations of sVEGFR-2, VEGF and bFGF were determined from blood samples taken at baseline, on day 14 of cycles 1, 2, 4, 6, etc. and at the final visit. Samples were analysed using the relevant quantitative enzyme-linked immunosorbent assay (ELISA) (R\&D Systems Europe, Oxford, UK) according to the manufacturer's instructions. DCE-MRI was performed at baseline, on day 2 (only cycle 1 ), and on day 14 of cycles $1-3$ to assess tumour blood flow/ tumour vessel permeability in a subgroup of patients (Morgan et al, 2003). 


\section{RESULTS}

\section{Patient characteristics}

A total of 71 patients (30 women, 41 men) with refractory advanced solid tumours were enroled into the BID noncontinuous and continuous treatment groups. Patients' median (range) age was $60(31-82)$ years, median (range) weight $73.6(39-113) \mathrm{kg}$. Baseline ECOG performance status was 0 in 36 patients (51\%), 1 in 32 patients $(45 \%)$ and 2 in 2 patients $(3 \%)$. For one patient, no baseline ECOG performance status was documented. The main tumour types were colorectal cancer $(n=25,35 \%)$, renal cell carcinoma $(n=12,17 \%)$, hepatocellular carcinoma $(n=9,13 \%)$, non-small cell lung cancer and pancreatic cancer (both $n=4,6 \%$ ). 56 patients (79\%) had prior systemic anticancer therapy, 63 (93\%) prior anticancer surgery, and $20(28 \%)$ prior radiotherapy. All 71 patients were valid for safety and pharmacokinetic analyses.

\section{Dose escalation and MTD}

Dose escalation started with a single oral dose of $10 \mathrm{mg}$ telatinib. The starting dose was based on nonclinical data. Based on the pharmacokinetic results of the first three patients, which showed a considerably lower than expected exposure, multiple dosing was initiated at $20 \mathrm{mg}$ OD. Doses of $20-300 \mathrm{mg}$ telatinib OD were administered for 14 days followed by 7 days off treatment. At doses of 150 and $300 \mathrm{mg}$ OD, no further increase in exposure to telatinib was achieved. As safety and tolerability remained good, BID dosing was initiated at $75 \mathrm{mg}$ BID using the same noncontinuous regimen of 14 days followed by 7 days off treatment. For the sake of clarity, the data presented in this paper refer to the patients enroled in the BID-dosing cohorts only. Up to $1500 \mathrm{mg}$ BID in the noncontinuous regimen, only two patients experienced DLTs, that is, grade 3 hypertension, at doses of 300 and $1500 \mathrm{mg}$ BID, respectively. A further dose escalation beyond the $1500 \mathrm{mg}$ BID dose level was not feasible due to the number of tablets to be taken. As the MTD had not been reached for the noncontinuous treatment, the continuous BID dosing was initiated at $600 \mathrm{mg}$ BID. Dose escalation was stopped at $1500 \mathrm{mg}$ BID continuous dosing without reaching the MTD of telatinib.

The results of the BID noncontinuous and continuous dosing groups are reported here. Telatinib was administered as solution and $25 \mathrm{mg}$ mesylate tablet in the $75 \mathrm{mg}$ BID noncontinuous dosing group, as solution, 25 and $150 \mathrm{mg}$ mesylate tablets, and $150 \mathrm{mg}$ base tablet in the $150 \mathrm{mg}$ BID noncontinuous dosing group, as $25 \mathrm{mg}$ mesylate tablet in the $300 \mathrm{mg}$ BID noncontinuous dosing group, and as $150 \mathrm{mg}$ tablet in all other groups. Table 1 shows an overview about the dose escalation steps and the treatment duration. Fifteen patients were enroled at the $150 \mathrm{mg}$ BID dose level as the relative bioavailability for different tablet formulations was evaluated at this dose level.

\section{Safety}

In total, $21 \%$ of all patients experienced at least one adverse event assessed by the investigators as study drug-related with worst CTC grade of $1-2$ and $25 \%$ at least one study drug-related adverse event with worst CTC grade 3 (Table 2). There were no study drugrelated adverse events of CTC grades 4 or 5 reported in this study.

The most common toxicity was hypertension in $4 \%$ of the patients with worst CTC grade 1-2 and in another $23 \%$ of the patients with worst CTC grade 3 (Table 2). Grade 3 hypertension occurred in one-third to half of the patients in the $600-1500 \mathrm{mg}$ noncontinuous dosing groups and the $900 \mathrm{mg}$ continuous dosing group (Table 2). In the $1200 \mathrm{mg}$ continuous dosing group, more than two-thirds of the patients experienced grade 3 hypertension. In most cases hypertension was clinically well manageable with a standard antihypertensive treatment. In three patients at dose
Table I Treatment summary by treatment group and dose level (all patients, $N=7$ I)

\begin{tabular}{lcc} 
Treatment group & $\mathbf{N}$ & $\begin{array}{c}\text { Duration (medication days) } \\
\text { (median (range) }\end{array}$ \\
\hline Noncontinuous dosing (I 4 days on/7 days off) & $106(35-182)$ \\
$75 \mathrm{mg}$ BID & 4 & $77(5-633)$ \\
$150 \mathrm{mg}$ BID & $15^{\mathrm{a}}$ & $58.5(14-287)$ \\
$300 \mathrm{mg}$ BID & 8 & $77.5(35-525)$ \\
$600 \mathrm{mg}$ BID & 6 & $156.5(55-497)$ \\
$900 \mathrm{mg}$ BID & 6 & $58.5(35-387)$ \\
$1500 \mathrm{mg}$ BID & 6 & \\
Continuous dosing & & $35(11-216)$ \\
$600 \mathrm{mg}$ BID & 7 & $120.5(64-164)$ \\
$900 \mathrm{mg}$ BID & 6 & $160(14-178)$ \\
$1200 \mathrm{mg}$ BID & 7 & $83(35-120)$ \\
$1500 \mathrm{mg}$ BID & 6 &
\end{tabular}

$\mathrm{BID}=$ two times daily. ${ }^{\mathrm{a}} \mathrm{At}$ the $150 \mathrm{mg} \mathrm{BID}$ dose level, the relative bioavailability of different tablet formulations was assessed. Therefore the data from 15 patients were pooled for this analysis.

levels of $300 \mathrm{mg}$ BID, $1500 \mathrm{mg}$ BID noncontinuous dosing and $1200 \mathrm{mg}$ BID continuous dosing, hypertension resulted in dose reduction and dose interruption, in one of them finally to permanent discontinuation of study drug treatment.

Other common adverse events were gastrointestinal toxicities such as anorexia and diarrhoea (Table 2). Diarrhoea led to dose reduction or interruption in four patients at dose levels of $900 \mathrm{mg}$ BID or higher, in one of them to permanent discontinuation. One patient at the $1500 \mathrm{mg}$ BID continuous dosing level had a dose interruption due to nausea and vomiting.

Serious study drug-related adverse events (adverse events leading to hospitalisation or assessed by the investigator as medically important) occurred in five patients: two patients had diarrhoea (dose levels: $1500 \mathrm{mg}$ BID noncontinuous and $1200 \mathrm{mg}$ BID continuous dosing), two patients had hypertension (dose levels: $1500 \mathrm{mg}$ BID noncontinuous and $1200 \mathrm{mg}$ BID continuous dosing), and one patient experienced a hand-foot skin reaction and dehydration (dose level: $900 \mathrm{mg}$ BID continuous dosing).

Dose-limiting toxicities were reported for two patients (dose levels: $300 \mathrm{mg}$ BID and $1500 \mathrm{mg}$ BID noncontinuous dosing). Both had hypertension refractory to standard treatment leading to dose reduction of telatinib. As at the highest dose level administered in this study, $1500 \mathrm{mg}$ BID continuous dosing, no patient out of six patients experienced dose-limiting toxicities within the first 21 days of treatment, the MTD was not reached in this study.

\section{Pharmacokinetics}

Day 14 steady-state geometric mean (percent coefficient of variation) telatinib and BAY 60-8246 pharmacokinetic parameters are shown in Table 3 and day 14 geometric mean telatinib plasma concentration $v s$ time profiles are shown in Figure 1. For the $150 \mathrm{mg}$ BID dose level, pharmacokinetic results were available from different exploratory formulations. For this dose level, results from only the $25 \mathrm{mg}$ telatinib mesylate tablet formulation are shown in Table 3 and Figure 1.

Following oral administration, telatinib was rapidly absorbed with median $t_{\max }$ of $3 \mathrm{~h}$ or less in the $75 \mathrm{mg}$ BID to $1500 \mathrm{mg}$ BID dose range. Geometric mean $C_{\max }$ increased in a less than doseproportional manner in the dose range of $75 \mathrm{mg}$ BID to $300 \mathrm{mg}$ BID. Geometric mean $C_{\max }$ increased two-fold between the 300 and $600 \mathrm{mg}$ BID dose level and subsequently increased in a less than dose-proportional manner up to $1500 \mathrm{mg}$ BID. Although a reason for the two-fold increase in geometric mean $C_{\max }$ is not known, it is not attributable to the $150 \mathrm{mg}$ telatinib mesylate tablet 
Table 2 Incidence of patients $(N \geqslant 2(\geqslant 3 \%))$ with study drug-related adverse events with worst CTC grades I -2 and incidence of patients with study drug-related adverse events with worst CTC grade $3^{\mathrm{a}}(\mathrm{N}(\%)$; all patients, $N=7 \mathrm{I})$

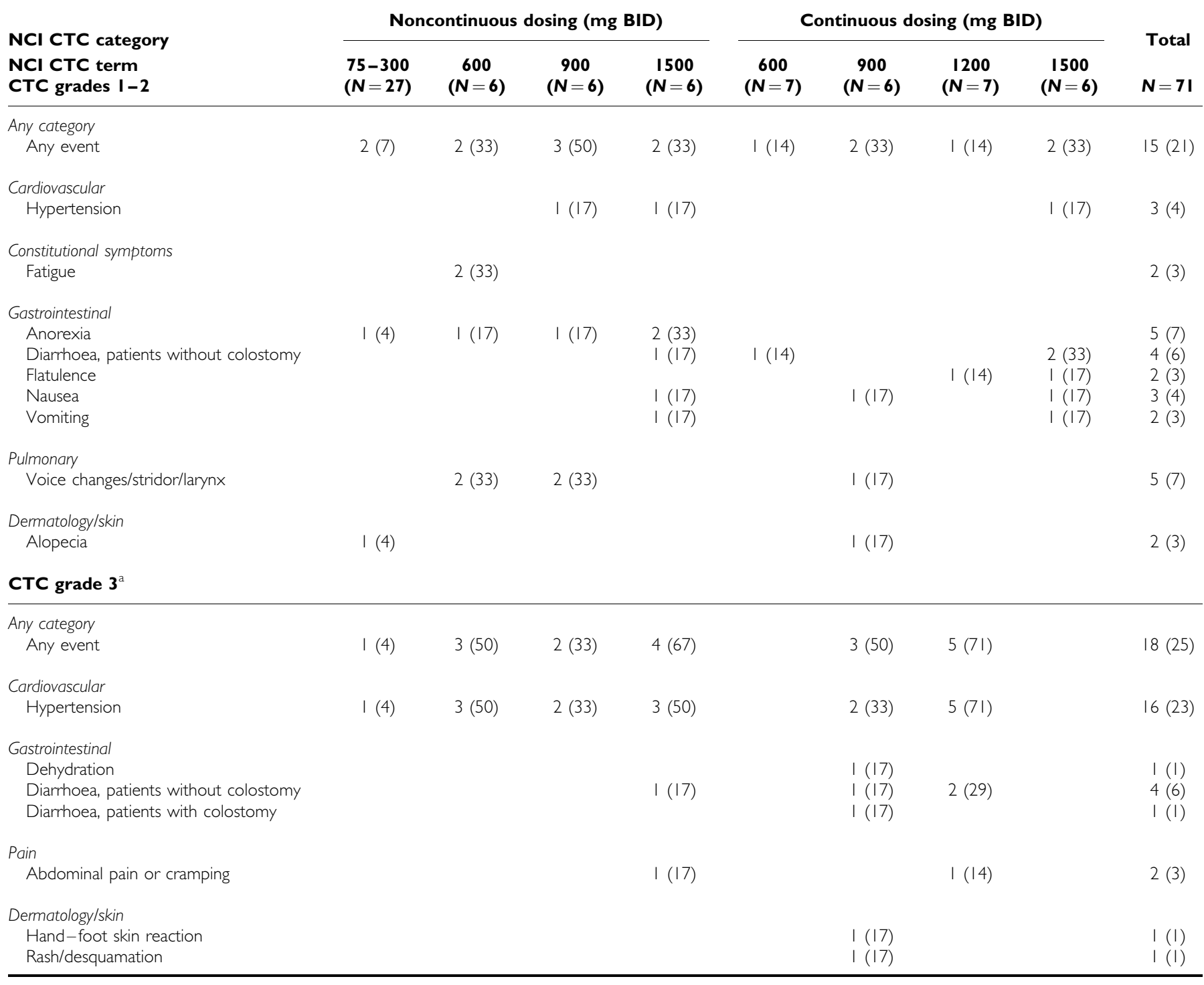

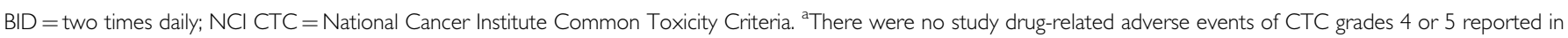
this study.

Table 3 Geometric mean (\%CV) day 14 telatinib and BAY 60-8246 pharmacokinetic parameters after oral administration of telatinib

\begin{tabular}{|c|c|c|c|c|c|c|c|}
\hline \multicolumn{8}{|l|}{ Telatinib } \\
\hline Dose & 75 mg BID & $150 \mathrm{mg}$ BID & 300 mg BID & $600 \mathrm{mg}$ BID & 900 mg BID & 1200 mg BID & $1500 \mathrm{mg}$ BID \\
\hline Mesylate tablet & $25 \mathrm{mg}$ & $25 \mathrm{mg}$ & $25 \mathrm{mg}$ & $150 \mathrm{mg}$ & $150 \mathrm{mg}$ & $150 \mathrm{mg}$ & $150 \mathrm{mg}$ \\
\hline$C_{\max }\left(\left.m g\right|^{-1}\right)$ & $0.212(140 \%)$ & $0.219(80 \%)$ & $0.389(153 \%)$ & $0.810(87 \%)$ & $1.275(57 \%)$ & $1.144(40 \%)$ & $0.964(83 \%)$ \\
\hline$A \cup C_{0-\operatorname{tn}}\left(\mathrm{mghl}^{-1}\right)$ & $1.38(150 \%)$ & $1.75(84 \%)$ & $1.92(142 \%)$ & $5.43(68 \%)$ & $7.41(51 \%)$ & $7.10(31 \%)$ & $6.17(80 \%)$ \\
\hline$A \cup C_{0-12}\left(\mathrm{mghl}^{-1}\right)$ & $1.39(146 \%)$ & $1.73(81 \%)$ & $2.86(176 \%)^{b}$ & $5.43(68 \%)$ & $7.30(52 \%)$ & 7.26 (31\%) & $6.29(84 \%)$ \\
\hline$t_{1 / 2}(h)$ & $7.4(33 \%)$ & $10.9(66 \%)^{\dagger}$ & $8.0(58 \%)^{b}$ & $8.7(46 \%)$ & $5.6(62 \%)^{b}$ & $5.6(100 \%)$ & $6.6(65 \%)$ \\
\hline$C_{\max }\left(\mathrm{mgl}^{-1}\right)$ & $0.02(202 \%)$ & $0.018(89 \%)$ & $0.021(133 \%)$ & $0.084(140 \%)$ & $0.216(74 \%)$ & $0.226(87 \%)$ & $0.102(147 \%)$ \\
\hline$t_{\max }(h)^{\mathrm{a}}$ & $3.0(0.5-6.0)$ & $2.5(1.0-12.3)$ & $1.5(0-8.5)$ & $2.1(0.6-10)$ & $3.7(1.0-8.6)$ & $4.0(2.1-4.3)$ & $3.5(0.5-6.1)$ \\
\hline$A \cup C_{0-\operatorname{tn}}\left(\mathrm{mghl}^{-1}\right)$ & $0.125(333 \%)^{c}$ & $0.157(74 \%)$ & $0.115(113 \%)^{b}$ & $0.650(128 \%)$ & $1.27(79 \%)$ & $1.66(81 \%)$ & $0.74(155 \%)$ \\
\hline$A \cup C_{0-12}\left(\mathrm{mghl}^{-1}\right)$ & $0.153(232 \%)$ & $0.155(72 \%)$ & $0.219(58 \%)^{b}$ & $0.650(128 \%)$ & $1.25(80 \%)$ & $1.71(84 \%)$ & $0.76(160 \%)$ \\
\hline
\end{tabular}

$\mathrm{AUC}_{0-12}=$ area under the plasma concentration versus time curve from time $0-12 \mathrm{~h} ; \mathrm{A} \cup \mathrm{C}_{0-\mathrm{tn}}=$ area under the plasma concentration versus time curve from time 0 to last data point; $\mathrm{BID}=$ two times daily; $C_{\max }=$ maximum plasma concentration; $\% \mathrm{CV}=$ percent coefficient of variation; $t_{\max }=$ time to reach maximum plasma concentration; $t_{1 / 2}=$ terminal half-life. ${ }^{\mathrm{a}}$ Median (range). ${ }^{\mathrm{b}}$ Sample size reduced by $2 .{ }^{\circ}$ Sample size reduced by 1 . 


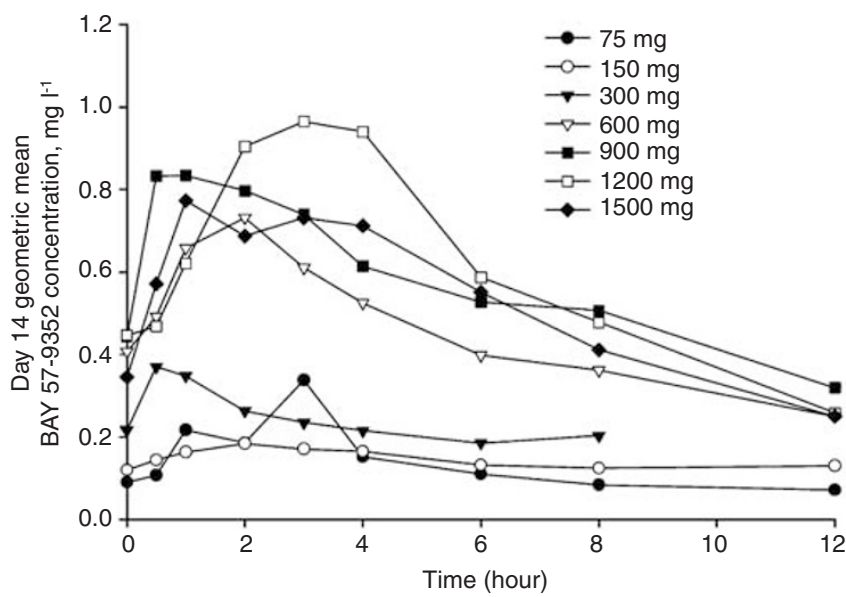

Figure I Geometric mean telatinib plasma concentration vs time profiles on day 14 of cycle 1.

formulation. Bioavailability assessments performed with the 25 and $150 \mathrm{mg}$ tablets indicated that the relative bioavailability of the $150 \mathrm{mg}$ tablet formulation is less when compared with the $25 \mathrm{mg}$ tablet formulation. Increases in telatinib geometric mean $\mathrm{AUC}_{0-\text { tn }}$ and $\mathrm{AUC}_{0-12}$ followed a pattern similar to that described for $C_{\max }$. Less than dose-proportional increase was observed in the $600-1500 \mathrm{mg}$ BID dose range. In general, exposure was comparable in the $900-1500 \mathrm{mg}$ BID dose range. These results (and relevant biomarker results) formed the basis for choosing $900 \mathrm{mg}$ BID as the recommended phase II dose for telatinib. The geometric mean half-life at the $900 \mathrm{mg}$ BID dose range was $5.6 \mathrm{~h}$ thus supporting the BID-dosing regimen.

After oral administration of telatinib, maximum concentrations of the metabolite (BAY 60-8246) were observed approximately around the same time as the parent compound or shortly thereafter. Plasma concentrations of BAY 60-8246 were generally lower when compared with telatinib plasma concentrations. At the recommended phase II dose of $900 \mathrm{mg}$ BID, geometric mean $C_{\max }$ and $\mathrm{AUC}_{0-12}$ values of the metabolite were less than $20 \%$ of the corresponding geometric mean $C_{\max }$ and $\mathrm{AUC}_{0-12}$ values of the parent compound. Less than dose-proportional increase observed with the parent compound was also observed with the metabolite. These results and the results of the mass balance study conducted in healthy subjects (data not shown) indicate that BAY $60-8246$ is of minor importance in humans.

\section{Pharmacodynamics}

To assess the biological activity of telatinib, plasma concentration analyses for the angiogenic markers VEGF, sVEGFR-2, bFGF, PDGF and IL- 6 were performed at baseline and during the course of the study. Furthermore, DCE-MRI measurements were done at baseline, on days 2 and 14 of cycle 1 and on day 14 of cycles 2 and 3. Evaluable DCE-MRI results were available for a subgroup of patients treated at dose levels of $300 \mathrm{mg}$ BID or higher.

VEGF plasma levels showed a dose-dependent short-term increase within $8 \mathrm{~h}$ after the first telatinib administration. VEGF levels increased also comparing day 21 to baseline. sVEGFR-2 levels showed a dose-dependent decrease over the course of the study. In addition, a decrease in the iAUC60 for the gadolinium curve as measured by DCE-MRI was observed.

The analysis of telatinib $\mathrm{AUC}_{0-12}$ on day 14 of cycle $1 \mathrm{vs}$ the ratio of gadolinium iAUC60 on day 14 of cycle 1 to iAUC60 at baseline is shown in Figure 2A. In general, the gadolinium iAUC60 ratio decreased with increasing telatinib $\mathrm{AUC}_{0-12}$ although a statistically significant correlation between telatinib exposure and the pharmacodynamic effect as measured by DCE-MRI was not observed (regression $r^{2}=0.0625$; Pearson correlation coefficient $\rho=-0.250$; test for no correlation, $\left.\mathrm{H}_{0}: \rho=0, P=0.10\right)$. Substantial decreases in the gadolinium iAUC60 ratio were observed at total daily doses of $\geqslant 600 \mathrm{mg}$ telatinib corresponding to telatinib $\mathrm{AUC}_{0-12}$ values of about $4 \mathrm{mg} \mathrm{h}^{-1}$.

The analysis of telatinib $\mathrm{AUC}_{0-12}$ on day 14 of cycle $1 v s$ the ratio of sVEGFR-2 in plasma on day 14 of cycle 1 to sVEGFR-2 at baseline is shown in Figure 2B. The ratio of sVEGFR-2 in plasma decreased with increasing telatinib $\mathrm{AUC}_{0-12}$, that is, essentially in an exposure-dependent manner (regression $r^{2}=0.2973$; Pearson correlation coefficient $\rho=-0.545$; test for no correlation, $\mathrm{H}_{0}$ : $\rho=0, P=0.0001$ ).

To correlate biomarker changes to the clinical outcome, the patients were categorised into those who had a progression-free survival of $<3$ months, 3 up to 6 months, or $>6$ months. The relative changes between cycle 1 , day 14 and baseline were calculated for VEGF, sVEGFR-2, bFGF, IL-8, tumour blood flow and tumour vessel permeability as measured by DCE-MRI and diastolic blood pressure (Figure 2C). Changes from baseline were observed for plasma VEGF and sVEGFR-2 levels, the decrease in tumour blood flow and permeability and also for the increase in diastolic blood pressure. The bFGF and IL-8 plasma levels showed no relevant changes after 14 days of multiple dosing with telatinib. The changes in VEGF and sVEGFR-2 plasma levels, the decrease in tumour blood flow and permeability and the increase in diastolic blood pressure were not predictive for the clinical outcome; there were no statistically significant differences in the change of biomarkers for patients who reached a progression-free survival of $>3$ months compared with those who stopped treatment during the first 3 months due to progressive disease.

\section{Efficacy}

Table 4 summarises the best tumour responses according to RECIST, study duration and medication days on telatinib by tumour type. Seventy-one patients were assessable for tumour response. Patients with renal cell carcinoma (RCC, $n=12$ ) showed the most promising preliminary antitumour activity: two of them reached a partial response, and the median treatment duration for patients with RCC was 164 days compared with 89 days in the overall study population.

\section{DISCUSSION}

Telatinib is safe and well tolerated up to doses of $1500 \mathrm{mg}$ BID continuous dosing. The most frequent study drug-related adverse events were hypertension and gastrointestinal toxicities such as anorexia and diarrhoea. The treatment with telatinib had to be dose reduced or discontinued permanently in only nine out of 71 patients due to drug-related averse events. At the highest dose level administered in this study, $1500 \mathrm{mg}$ BID continuous dosing, none of the six patients experienced a DLT within the first 21 days of treatment, whereas at $1500 \mathrm{mg}$ BID noncontinuous dosing, one out of six patients experienced a DLT, that is, grade 3 hypertension refractory to standard treatment. The MTD was not reached in this study.

The safety profile of telatinib is comparable to other smallmolecule VEGFR-inhibiting compounds. Hypertension as a common class toxicity phenomenon was clinically well manageable in most of the patients with a standard antihypertensive treatment. Recently, Steeghs et al (2008) reported that small vessel rarefaction may be one of the underlying haemodynamic mechanisms causing hypertension. The average increase in diastolic blood pressure in our study was comparable to those reported results.

In our study, diarrhoea led to dose reductions in three patients. The occurrence of gastrointestinal toxicities is also known for 
A
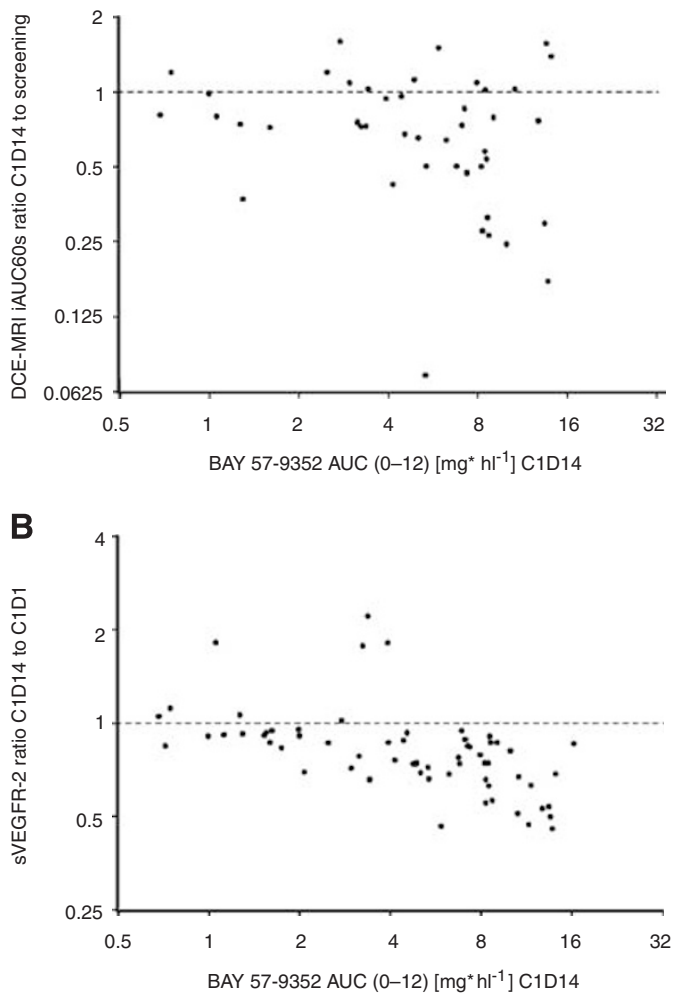

C
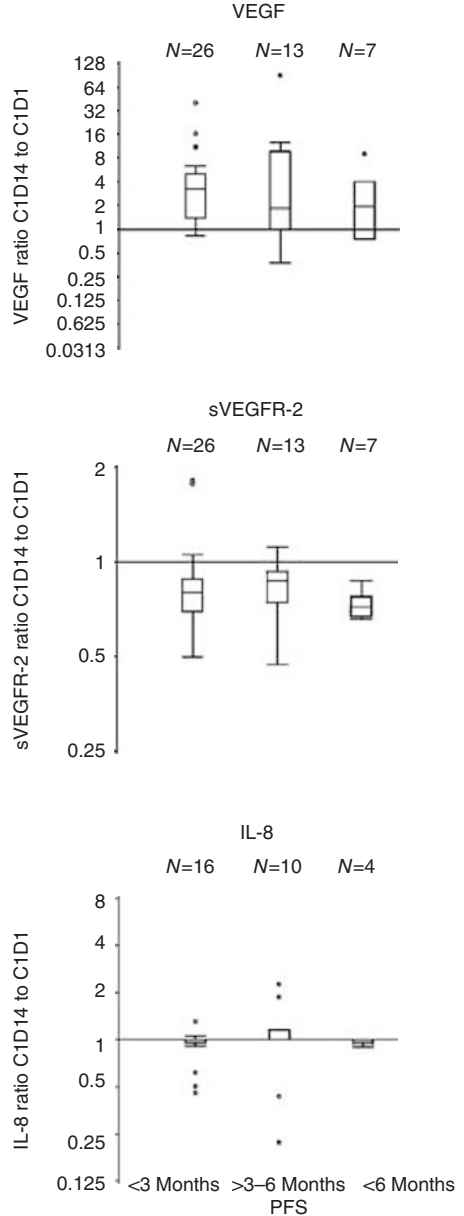
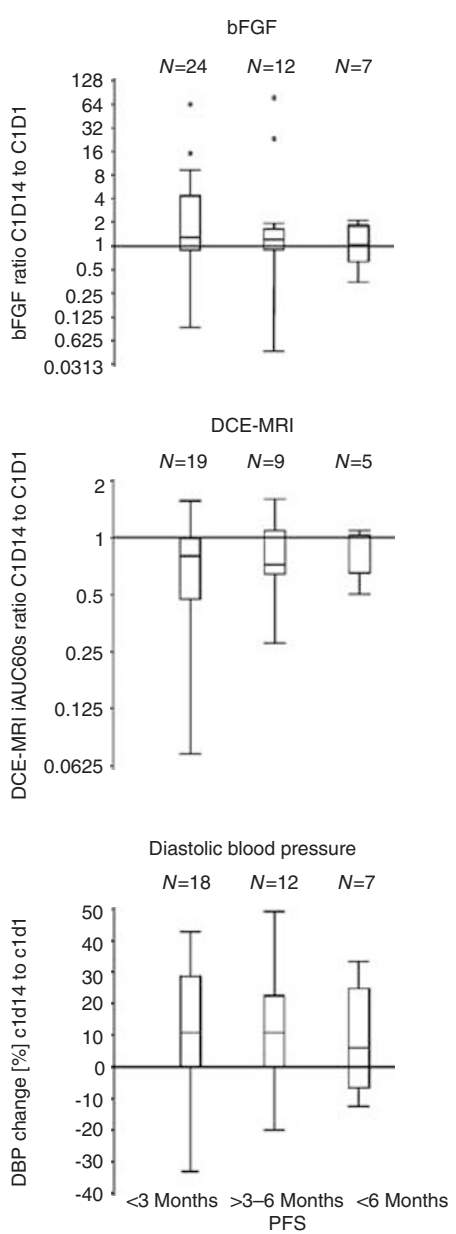

Figure 2 Analysis of telatinib $A_{U} C_{0-12}$ on day 14 of cycle I vs the ratio of the initial 60 s area under the gadolinium curve (iAUC60) on day I4 of cycle I to the iAUC60 at baseline (A) and vs the ratio of sVEGFR-2 in plasma on day I 4 of cycle I to sVEGFR-2 at baseline (B); correlation of pharmacodynamic parameters to progression-free survival $(\mathbf{C})$

Table 4 Best response by tumour type and overall response according to RECIST

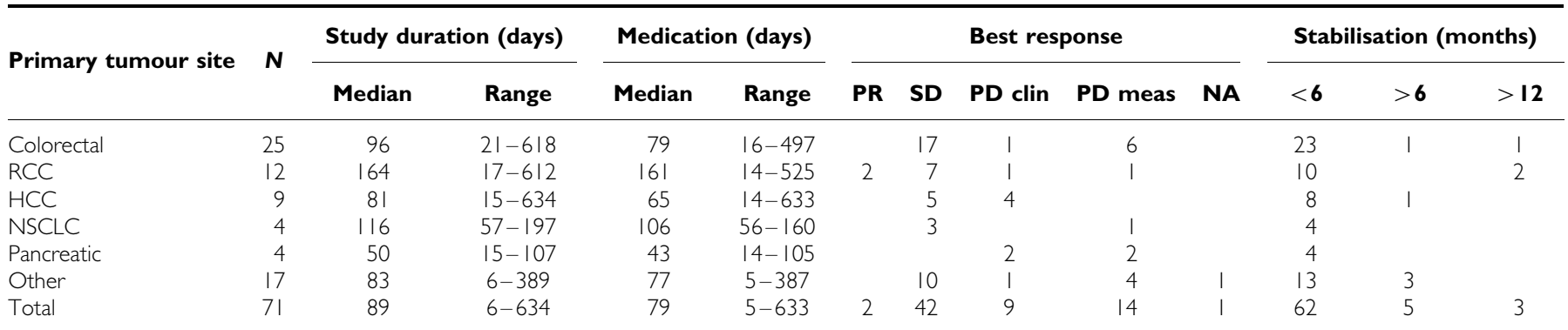

$\mathrm{HCC}=$ hepatocellular carcinoma; $\mathrm{NA}=$ not assessable for response; $\mathrm{NSCLC}=$ non-small-cell lung cancer; $\mathrm{PD}$ clin=progressive disease, clinical judgement; $\mathrm{PD}$ meas = progressive disease, measurement proven; $\mathrm{PR}=$ partial response; $\mathrm{RCC}=$ renal cell carcinoma; $\mathrm{RECIST}=$ Response Evaluation Criteria in Solid Tumours; $\mathrm{SD}=$ stable disease.

other VEGF-inhibiting compounds (Escudier et al, 2007; Motzer et al, 2007). The variability of pharmacokinetic parameters was significant. Geometric mean exposure to telatinib increased in a less than dose-proportional manner up to $1500 \mathrm{mg}$ BID. In general, exposure was similar in the $900-1500 \mathrm{mg}$ BID dose range. Thus further increase in dose did not result in a further increase in drug exposure. The short half-life of $6.6-10.9 \mathrm{~h}$ was the reason for BID administration of telatinib.

The biomarkers assessed in this study demonstrated the biological activity of telatinib. The angiogenic factors VEGF and sVEGFR-2 showed effects known from other VEGF-inhibiting compounds. Increases in VEGF and decreases in sVEGFR-2 were dose-dependent and correlated to telatinib exposure. The DCEMRI parameters Ktrans and iAUC60 showed a proof of mechanism for telatinib. However, there was no correlation between the clinical outcome and the biomarker activity. This might be due to the heterogeneous study population and the various dose levels used in this study.

The safety profile of telatinib was acceptable and a toxic dose level with two out of six or more DLTs at one dose level was not reached in this study even at the highest dose of $1500 \mathrm{mg}$ BID continuously administered. A further dose escalation was not 
feasible due to the number of tablets to be taken at these high dose levels and the pharmacokinetic data showed that an exposure plateau was reached at dose levels of $900 \mathrm{mg}$ BID or higher. In concordance with the pharmacokinetic exposure, the pharmacodynamic data revealed no additional effects beyond the $900 \mathrm{mg}$ BID dose level. Taking the tolerability, pharmacokinetic and biomarker data into consideration, the recommended phase II dose level for single-agent telatinib is $900 \mathrm{mg}$ BID administered continuously.

The treatment with telatinib showed anticancer effects in two patients with RCC who reached a partial remission. RCC is one of the tumour types most sensitive to VEGF-inhibiting therapeutics (Escudier et al, 2007; Motzer et al, 2007).

In conclusion, telatinib is an orally available small-molecule inhibitor of VEGFR-2/-3 and PDGFR- $\beta$ tyrosine kinases with a favourable safety profile in patients with refractory advanced solid tumours. The observed antitumour activity and pharmacodynamic results warrant further evaluation of telatinib in patients with advanced cancer. The recommended phase II dose of telatinib is $900 \mathrm{mg}$ BID as continuous dosing based on pharmacokinetic data, the toxicity profile and the biomarker evaluations.

\section{REFERENCES}

Algire GH, Chalkley HW, Legallais FY, Park HD (1945) Vascular reactions of normal and malignant tissues in vivo. I. Vascular reactions of mice to wounds and to normal and neoplastic transplants. J Natl Cancer Inst 6: $73-85$

Asano M, Yukita A, Matsumoto T, Kondo S, Suzuki H (1995) Inhibition of tumor growth and metastasis by an immunoneutralizing monoclonal antibody to human vascular endothelial growth factor/vascular permeability factor 121. Cancer Res 55: 5296-5301

Borgstrom P, Bourdon MA, Hillan KJ, Sriramarao P, Ferrara N (1998) Neutralizing anti-vascular endothelial growth factor antibody completely inhibits angiogenesis and growth of human prostate carcinoma micro tumors in vivo. Prostate 35: $1-10$

Dosquet C, Coudert MC, Lepage E, Cabane J, Richard F (1997) Are angiogenic factors, cytokines, and soluble adhesion molecules prognostic factors in patients with renal cell carcinoma? Clin Cancer Res 3: $2451-2458$

Ellis LM (2004) Angiogenesis and its role in colorectal tumor and metastasis formation. Semin Oncol 31: 3-9

Escudier B, Eisen T, Stadler WM, Szczylik C, Oudard S, Siebels M, Negrier S, Chevreau C, Solska E, Desai AA, Rolland F, Demkow T, Hutson TE, Gore M, Freeman S, Schwartz B, Shan M, Simantov R, Bukowski RM (2007) Sorafenib in advanced clear-cell renal-cell carcinoma. $N$ Engl J Med 356: $125-134$

Folkman J (1971) Tumor angiogenesis: therapeutic implications. N Engl J Med 285: $1182-1186$

Folkman J (1990) What is the evidence that tumors are angiogenesis dependent? J Natl Cancer Inst 82: 4-6

Folkman J (2002) Role of angiogenesis in tumor growth and metastasis. Semin Oncol 29: $15-18$

Giatromanolaki A, Koukourakis MI, Turley H, Sivridis E, Harris AL, Gatter KC (2006) Phosphorylated KDR expression in endometrial cancer cells relates to HIF1alpha/VEGF pathway and unfavourable prognosis. Mod Pathol 19: 701 - 707

Goldman CK, Kendall RL, Cabrera G, Soroceanu L, Heike Y, Gillespie GY, Siegal GP, Mao X, Bett AJ, Huckle WR, Thomas KA, Curiel DT (1998) Paracrine expression of a native soluble vascular endothelial growth factor receptor inhibits tumor growth, metastasis, and mortality rate. Proc Natl Acad Sci USA 95: 8795-8800

Kim KJ, Li B, Winer J, Armanini M, Gillett N, Phillips HS, Ferrara N (1993) Inhibition of vascular endothelial growth factor-induced angiogenesis suppresses tumour growth in vivo. Nature 362: $841-844$

Melnyk O, Shuman MA, Kim KJ (1996) Vascular endothelial growth factor promotes tumor dissemination by a mechanism distinct from its effect on primary tumor growth. Cancer Res 56: $921-924$

Millauer B, Longhi MP, Plate KH, Shawver LK, Risau W, Ullrich A, Strawn LM (1996) Dominant-negative inhibition of Flk-1 suppresses the growth of many tumor types in vivo. Cancer Res 56: 1615-1620

Morgan B, Thomas AL, Drevs J, Hennig J, Buchert M, Jivan A, Horsfield MA, Mross K, Ball HA, Lee L, Mietlowski W, Fuxuis S, Unger C, O’Byrne K, Henry A, Cherryman GR, Laurent D, Dugan M, Marme D, Steward WP (2003) Dynamic contrast-enhanced magnetic resonance imaging as a biomarker for the pharmacological response of PTK787/ZK 222584 , an inhibitor of the vascular endothelial growth factor receptor tyrosine kinases, in patients with advanced colorectal cancer and liver metastases: results from two phase I studies. J Clin Oncol 21: 3955 - 3964

Motzer RJ, Hutson TE, Tomczak P, Michaelson MD, Bukowski RM, Rixe O, Oudard S, Negrier S, Szczylik C, Kim ST, Chen I, Bycott PW, Baum CM, Figlin RA (2007) Sunitinib $v s$ interferon alfa in metastatic renal-cell carcinoma. $N$ Engl J Med 356: $115-124$

Pisacane AM, Risio M (2005) VEGF and VEGFR-2 immunohistochemistry in human melanocytic naevi and cutaneous melanomas. Melanoma Res 15: $39-43$

Raben D, Helfrich B (2004) Angiogenesis inhibitors: a rational strategy for radiosensitization in the treatment of non-small-cell lung cancer? Clin Lung Cancer 6: 48-57

Steeghs N, Gelderblom H, Roodt JO, Christensen O, Rajagopalan P, Hovens M, Putter H, Rabelink TJ, de Koning E (2008) Hypertension and rarefaction during treatment with telatinib, a small molecule angiogenesis inhibitor. Clin Cancer Res 14: $3470-3476$

Therasse P, Arbuck SG, Eisenhauer EA, Wanders J, Kaplan RS, Rubinstein L, Verweij J, Van Glabbeke M, van Oosterom AT, Christian MC, Gwyther SG (2000) New guidelines to evaluate the response to treatment in solid tumors. European Organization for Research and Treatment of Cancer, National Cancer Institute of the United States, National Cancer Institute of Canada. J Natl Cancer Inst 92: 205-216

Trotti A, Byhardt R, Stetz J, Gwede C, Corn B, Fu K, Gunderson L, McCormick B, Morrisintegral M, Rich T, Shipley W, Curran W (2000) Common toxicity criteria: version 2.0. An improved reference for grading the acute effects of cancer treatment: impact on radiotherapy. Int J Radiat Oncol Biol Phys 47: 13-47

Warren RS, Yuan H, Matli MR, Gillett NA, Ferrara N (1995) Regulation by vascular endothelial growth factor of human colon cancer tumorigenesis in a mouse model of experimental liver metastasis. J Clin Invest 95: $1789-1797$

Wedge SR, Ogilvie DJ, Dukes M, Kendrew J, Chester R, Jackson JA, Boffey SJ, Valentine PJ, Curwen JO, Musgrove HL, Graham GA, Hughes GD, Thomas AP, Stokes ES, Curry B, Richmond GH, Wadsworth PF, Bigley AL, Hennequin LF (2002) ZD6474 inhibits vascular endothelial growth factor signaling, angiogenesis, and tumor growth following oral administration. Cancer Res 62: $4645-4655$

Wood JM, Bold G, Buchdunger E, Cozens R, Ferrari S, Frei J, Hofmann F, Mestan J, Mett H, O’Reilly T, Persohn E, Rosel J, Schnell C, Stover D, Theuer A, Towbin H, Wenger F, Woods-Cook K, Menrad A, Siemeister G, Schirner M, Thierauch KH, Schneider MR, Drevs J, Martiny-Baron G, Totzke F (2000) PTK787/ZK 222584, a novel and potent inhibitor of vascular endothelial growth factor receptor tyrosine kinases, impairs vascular endothelial growth factor-induced responses and tumor growth after oral administration. Cancer Res 60: 2178-2189

Yuan F, Chen Y, Dellian M, Safabakhsh N, Ferrara N, Jain RK (1996) Timedependent vascular regression and permeability changes in established human tumor xenografts induced by an anti-vascular endothelial growth factor/vascular permeability factor antibody. Proc Natl Acad Sci USA 93: $14765-14770$

Zhang HT, Craft P, Scott PA, Ziche M, Weich HA, Harris AL, Bicknell R (1995) Enhancement of tumor growth and vascular density by transfection of vascular endothelial cell growth factor into MCF-7 human breast carcinoma cells. J Natl Cancer Inst 87: 213-219 